\title{
Complications after mesial temporal lobe surgery via inferior temporal gyrus approach
}

\author{
Fernando L. Vale, M.D., ${ }^{1}$ Stephen Reintjes, M.D., ${ }^{1}$ And Hermes G. Garcia, M.D. ${ }^{2}$ \\ ${ }^{1}$ Department of Neurosurgery and Brain Repair, University of South Florida, Tampa, Florida; and \\ ${ }^{2}$ Department of Neurosurgery, University of Puerto Rico, San Juan, Puerto Rico
}

Object. The purpose of this study was to identify the complications associated with the inferior temporal gyrus approach to anterior mesial temporal lobe resection for temporal lobe epilepsy.

Methods. This retrospective study examined complications experienced by 483 patients during the 3 months after surgery. All surgeries were performed during 1998-2012 by the senior author (F.L.V.).

Results. A total of 13 complications $(2.7 \%)$ were reported. Complications were 8 delayed subdural hematomas $(1.6 \%), 2$ superficial wound infections $(0.4 \%), 1$ delayed intracranial hemorrhage $(0.2 \%), 1$ small lacunar stroke $(0.2 \%)$, and 1 transient frontalis nerve palsy $(0.2 \%)$. Three patients with subdural hematoma $(0.6 \%)$ required readmission and surgical intervention. One patient $(0.2 \%)$ with delayed intracranial hemorrhage required readmission to the neuroscience intensive care unit for observation. No deaths or severe neurological impairments were reported. Among the 8 patients with subdural hematoma, 7 were older than 40 years $(87.5 \%)$; however, this finding was not statistically significant $(\mathrm{p}=0.198)$.

Conclusions. The inferior temporal gyrus approach to mesial temporal lobe resection is a safe and effective method for treating temporal lobe epilepsy. Morbidity and mortality rates associated with this procedure are lower than those associated with other neurosurgical procedures. The finding that surgical complications seem to be more common among older patients emphasizes the need for early surgical referral of patients with medically refractory epilepsy.

(http://thejns.org/doi/abs/10.3171/2013.3.FOCUS1354)

KEY WoRds
pathology

$\mathrm{M}$ ESIAL temporal lobe epilepsy is usually resistant to medical management; ${ }^{8}$ for those resistant cases, surgical intervention has been proven to be effective. ${ }^{20,25}$ The aim of surgery is resection of the mesial structures of the temporal lobe with minimal complications and no deaths. Although several studies of MTLE surgery indicate that seizures are resolved for as many as $75 \%-90 \%$ of adequately chosen candidates, ${ }^{12,14,19,25}$ misconceptions and stigmas surrounding epilepsy surgery prevent referral of some patients for early treatment. ${ }^{4}$

Schemes for mesial temporal lobe surgery have evolved over the past 20 years. Approaches, exposures, and corridors have changed as neuroimaging, surgical

Abbreviations used in this paper: MTLE = mesial temporal lobe epilepsy; ITG = inferior temporal gyrus. navigation systems, and knowledge of temporal lobe anatomy have improved. Each approach has its own advantages and consequences. In addition, recent advances in technology have resulted in multimodal approaches to the control of epilepsy, such as Gamma Knife radiosurgery ${ }^{1,2}$ and laser ablation therapy. ${ }^{6}$

Over the past 14 years, refinements have been made to temporal lobe resection and amygdalohippocampectomy with the ITG approach. ${ }^{7,21}$ This approach involves gaining selective exposure by creating a small craniotomy with a cortical trajectory through the ITG. We describe the postsurgical complications that can occur when this approach is used to treat mesial temporal lobe pathology. These results should help with quantification of the risks and complications of this technique and should be useful for evaluation of various surgical modalities of treatment for medically refractory MTLE. 


\section{F. L. Vale, S. Reintjes, and H. G. Garcia}

\section{Methods}

This report was approved by the institutional review board, and patients provided informed consent.

Since 1998, a prospective database has been established for all patients surgically treated for medically refractory epilepsy at the University of South Florida. This study included all patients who underwent mesial temporal lobe resection via an inferior temporal gyrus corridor and who agreed to at least 6 months of follow-up. Before surgery, all patients underwent a standardized preoperative workup that included long-term video electroencephalography monitoring, high-resolution 1.5-T or 3-T MRI with attention to temporal lobes, PET/SPECT, and the Wada test. Indication for surgery and side of surgery were determined by the above workup and discussion among neurosurgeon, neurologist, and neuroradiologist. This collection of data was approved by the university hospital's institutional review board and was performed in accordance with requirements of the Health Insurance Portability and Accountability Act of 1996.

The data analyzed included patient sex, age at surgery, side of surgery, and MRI findings before surgery. Patients with the following MRI findings were included in this study: presence or absence of mesial temporal sclerosis, benign brain tumors, vascular lesions, and other/unknown conditions (revisions of surgeries previously performed at another institution). Patients with the following were excluded from this study: high-grade gliomas, cerebral metastasis, and new-onset seizures. Postoperative scans and neurological examination were used to document complications secondary to surgery. Formal visual field testing was not routinely performed postoperatively, but bedside testing results were routinely documented.

The surgery performed for all patients was selective anterior mesial lobe resection and/or lesionectomy via a transcortical inferior temporal gyrus approach (Fig. 1). ${ }^{7,21}$ The surgical technique has been described in detail and involves a small temporal trephine craniotomy with exposure limited to the middle and inferior temporal gyri. A 6- to 8-cm vertical incision 1 fingerbreadth anterior to the tragus was made. Because the anterior temporal lobe resection was not extensive, exposure to the superior temporal gyrus, sylvian fissure, or frontal lobes was avoided. The goal of surgery was to remove the hippocampus and surrounding structures (parahippocampus) to the level of the superior colliculi through a corticectomy at the level of the ITG. Complete resection of the uncus and partial resection (more than $80 \%$ ) of the amygdala were also performed. If a lesion (tumor or cavernous malformation) was present, it was resected. All lesions were limited to the anteromesial temporal lobe. Neuronavigation was routinely used for these cases. Intravenous cefazolin and $10 \mathrm{mg}$ of intravenous dexamethasone were given at the start of the operation. Mannitol was given as needed for brain relaxation (25-50 g; single dose). Mild hypocapnia $\left(\mathrm{PCO}_{2} 28-32 \mathrm{~mm} \mathrm{Hg}\right)$ was routinely induced at the beginning of the intervention, but $\mathrm{PCO}_{2}$ was normalized after CSF drainage was achieved. Dural closure was intended to be watertight; dural sealant was added as necessary. The senior author (F.L.V.) performed all surgeries. After

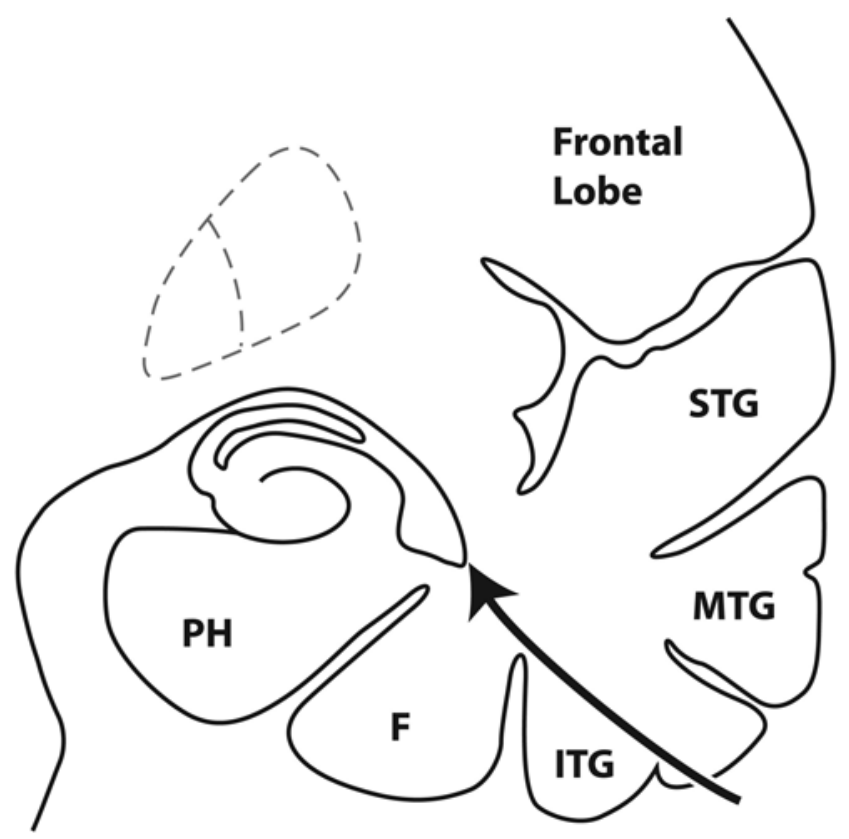

FIG. 1. Schematic diagram of the ITG approach to mesial structures. Coronal cut of the temporal lobe at the level of the zygomatic root. Arrow represents the trajectory for temporal horn exposure. $F=$ fusiform gyrus; $\mathrm{MTG}=$ middle temporal gyrus; $\mathrm{PH}=$ parahippocampal gyrus; STG = superior temporal gyrus.

surgery, all patients were monitored in the neuroscience intensive care unit, postoperative head CT was performed within 24 hours, and complete neurological assessment and follow-up brain MRI were conducted at 3 months. No patient was lost to follow-up.

Statistical analyses were performed to identify factors that influence rates of complication. Data were stored on an Excel spreadsheet. For calculations involving age, logistic regression (2-tailed) analyses were conducted by using the $\mathrm{R}$ statistical computing program. For calculations not involving patient age, VassarStats was used to conduct a 2-tailed Fisher exact test. Alpha was set to 0.05 .

\section{Results}

A total of 483 eligible patients who had undergone selective anterior mesial temporal lobe resection through an ITG approach for MTLE (confirmed through a standardized protocol) were identified. The mean patient age was 36.7 years (youngest 4 years and oldest 75 years); 166 patients $(34.3 \%)$ were younger than $30,121(25.0 \%)$ were $30-39,121(25.0 \%)$ were $40-49,58(12.0 \%)$ were $50-59$, and $17(3.5 \%)$ were older than 60 years of age (Table 1$)$. Of the 483 patients, $247(48.9 \%)$ were female and 236 (51.1\%) were male. Brain MR images showed no lesions in 180 patients $(37.3 \%)$; mesial temporal sclerosis was found in 233 patients (48.2\%), a benign brain tumor lesion in 29 (6.0\%), cavernous hemangioma in 10 (2.07\%), and unknown pathology in $31(6.42 \%)$ (first surgery was performed at another institution and limited documentation was available).

A total of 13 complications $(2.7 \%)$ were associated with this surgical approach (Table 2). The complications 
Complications from mesial temporal lobe surgery

TABLE 1: Characteristics of 483 patients who underwent mesial temporal lobe surgery via an inferior temporal gyrus approach* $^{*}$

\begin{tabular}{|c|c|c|c|}
\hline \multirow[b]{2}{*}{ Characteristic } & \multirow[b]{2}{*}{ No. $(\%)$} & \multicolumn{2}{|c|}{ 2-Tailed $p$ Value } \\
\hline & & Fisher Exact Test & Logistic Regression \\
\hline \multicolumn{4}{|l|}{ sex } \\
\hline$F$ & $247(48.9)$ & 0.40 & NA \\
\hline$M$ & $236(51.1)$ & & \\
\hline \multicolumn{4}{|l|}{ age category in yrs } \\
\hline older than 30 & $317(65.6)$ & NA & 0.13 \\
\hline older than 40 & $196(40.6)$ & NA & 0.19 \\
\hline older than 50 & $75(15.5)$ & NA & 0.43 \\
\hline older than 60 & $17(3.5)$ & NA & 0.49 \\
\hline preop MRI finding & & & NA \\
\hline MTS & $233(48.2)$ & 0.58 & NA \\
\hline no lesion & $180(37.3)$ & 1.00 & NA \\
\hline benign tumors & $29(6.0)$ & 0.04 & NA \\
\hline other & $31(6.42)$ & 0.62 & NA \\
\hline $\mathrm{CH}$ & $10(2.07)$ & 1.00 & NA \\
\hline side of resection & & & NA \\
\hline $\mathrm{rt}$ & $250(51.8)$ & 0.78 & NA \\
\hline It & $233(48.2)$ & & \\
\hline
\end{tabular}

* $\mathrm{CH}=$ cavernous hemangioma; MTS = mesial temporal sclerosis; NA = not applicable.

were 8 subdural hematomas $(1.6 \%), 2$ superficial wound infections $(0.4 \%), 1$ delayed intracranial hemorrhage $(0.2 \%), 1$ small lacunar stroke $(0.2 \%)$, and 1 case of partial frontalis nerve palsy $(0.2 \%)$. Three patients $(0.6 \%)$ with subdural hematoma required readmission to the hospital and surgical intervention. One patient with delayed intracranial hemorrhage in the surgical site was readmitted to the neuroscience intensive care unit for observation. No deaths or severe neurological impairment were reported.

Overall, the most common complication was delayed subdural hematoma. Of the 8 patients with subdural hematoma, 6 had persistent headaches, 1 had transient confusion, and 1 patient was asymptomatic (subdural hematoma discovered during the 3-month follow-up MRI). All subdural hematomas were located at the cranial convexity, ipsilateral to the surgery site (Fig. 2). Of these 8 patients, 7 had chronic subdural hematoma and 1 had subacute subdural fluid collection; 7 (87.5\%) were older than 40 years. Three patients, who had experienced mass effect and persistent symptoms, underwent surgical removal of the subdural hematoma and were sent home without neurological deficit.

The second most common complication was superficial wound infection. Of the 2 patients $(0.4 \%)$ with wound infection, 1 underwent wound revision and both required long-term antibiotics. Both wounds healed quickly and without long-term disfigurement.

Complications did not differ significantly among groups. Fisher exact test indicated no significant difference in complication rate between male and female patients $(\mathrm{p}=0.40)$ or between left-sided and right-sided pathology $(p=0.78)$. Preoperative MRI findings also did not play a role in the surgical complication rate, unless the pathology included a benign tumor $(\mathrm{p}=0.04)$. However, we believe that this finding most likely represents a Type 1 error because of the small number of tumor cases in this series. Other radiographic findings that did not contribute to the rate of complications were cavernomas $(\mathrm{p}=1.0)$, mesial temporal sclerosis $(\mathrm{p}=0.57)$, no lesions on $\mathrm{MR}$ images $(\mathrm{p}=1.0)$, and other $(\mathrm{p}=0.61)$.

Although most complications occurred in patients older than $30(76.9 \%)$ and $40(61.5 \%)$ years, age did not contribute significantly to the complications ( $p=0.134$ and $\mathrm{p}=0.193$, respectively). The rate of complications was not statistically significant for those in the oldest age groups, older than $50(\mathrm{p}=0.43)$ and older than 60 years $(\mathrm{p}=0.486)$.

\section{Discussion}

Surgery is an effective treatment for medically refractory epilepsy. ${ }^{25}$ Among patients with MTLE and mesial temporal sclerosis, surgery reduced seizures for approximately $75 \% ., 12,19,23$ Although this surgery is considered safe and effective, it is not free of complications. Surgical intervention has changed and will continue to change for the foreseeable future because of advances in neuroimaging, neuronavigation, and anesthesia. In addition, newer techniques have expanded the surgical armamentarium of epilepsy surgeons. When recommending surgery, it is necessary to understand and quantify the risks and complications associated with each technique. For a more accurate representation, surgical outcomes and complications need to be completely reevaluated.

The transcortical approach is a known surgical technique for treating mesial temporal pathology. Historically, the approach has required extensive neocorti- 
F. L. Vale, S. Reintjes, and H. G. Garcia

TABLE 2: Complications associated with mesial temporal lobe resection via inferior temporal gyrus approach*

\begin{tabular}{|c|c|c|c|c|c|c|c|}
\hline Case No. & Age (yrs), Sex & Side of Resection & Preop MRI Finding & Complication & Readmission & Reop & Permanent Deficit \\
\hline 1 & $18, \mathrm{M}$ & It & tumor & wound infection & yes & no & no \\
\hline 2 & $20, F$ & $r t$ & tumor & palsy & no & no & no \\
\hline 3 & $29, \mathrm{~F}$ & $r t$ & no lesion & SDH & no & no & no \\
\hline 4 & $33, \mathrm{~F}$ & $\mathrm{rt}$ & MTS & wound infection & yes & yes & no \\
\hline 5 & 40. F & $\mathrm{rt}$ & no lesion & $\mathrm{SDH}$ & no & no & no \\
\hline 6 & $44, \mathrm{M}$ & It & normal & $\mathrm{SDH}$ & yes & yes & no \\
\hline 7 & $55, M$ & It & MTS & $\mathrm{SDH}$ & yes & yes & no \\
\hline 8 & $55, M$ & It & tumor & $\mathrm{SDH}$ & yes & yes & no \\
\hline 9 & $55, M$ & It & MTS & $\mathrm{SDH}$ & no & no & no \\
\hline 10 & $55, M$ & It & MTS & $\mathrm{ICH}$ & yes & no & no \\
\hline 11 & $56, M$ & It & no lesion & $\mathrm{SDH}$ & no & no & no \\
\hline 12 & $59, \mathrm{~F}$ & $r t$ & no lesion & $\mathrm{SDH}$ & no & no & no \\
\hline 13 & $64, \mathrm{~F}$ & It & MTS & lacunar stroke & no & no & yes \\
\hline
\end{tabular}

* $\mathrm{ICH}=$ intracranial hemorrhage; MTS = mesial temporal sclerosis; palsy = partial frontalis nerve palsy; $\mathrm{SDH}=$ subdural hematoma.

cal resection, which results in a unique set of potential complications, especially pathology of the dominant temporal lobe. With the goal of minimizing complications and hastening patient recovery, less invasive techniques have been sought. Technologic advances have made less aggressive resections possible. ${ }^{17,19}$ Access to the mesial temporal lobe structures through an ITG approach have been described ${ }^{7,21}$ and have resulted in long-term seizure control for approximately $80 \%$ of patients with mesial temporal sclerosis ${ }^{12}$ and $55 \%$ with no lesions on MR images. ${ }^{22}$ Understanding the complications of this technique is imperative for education of patients and caregivers.
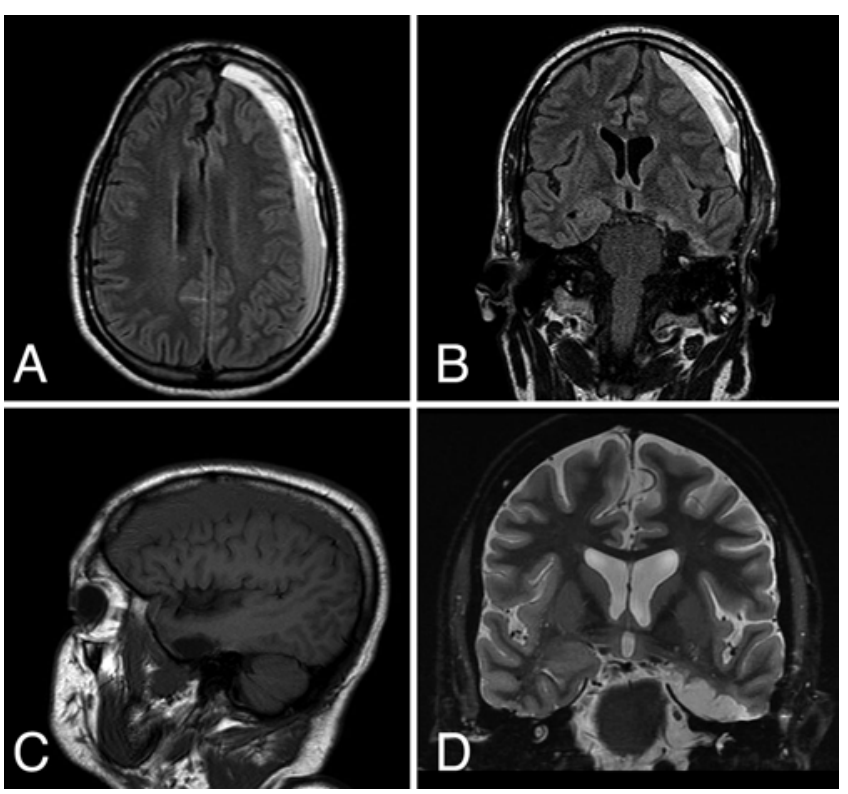

FIG. 2. MR images demonstrating a convexity subdural hematoma after mesial temporal lobe resection. A and B: Axial and coronal FLAIR sequences demonstrating subdural hematoma. C: Sagittal T1-weighted image depicting chronic subdural hematoma. D: Coronal T2-weighted image obtained 2 months after evacuation of subdural hematoma.
The total complication rate for our technique was $2.7 \%$, which compares favorably with the currently reported $2.9 \%-8.4 \%{ }^{3,13,14,16,18}$ Differences in surgical techniques, pathology, and categorization make these results difficult to compare. Complications are usually categorized as surgical or neurological but sometimes as major or minor. The type of complications that cause the most fear and concern for patients and their families are the neurological complications. Our rate of permanent neurological complications was $0.2 \%$, which is analogous to other documented rates $(0.1 \%-2.0 \%))^{3,13,16,18}$

The most common permanent neurological complication described in the literature is hemiparesis. Major hemiparesis was not reported in our series of MTLE cases, probably because our surgical route does not require manipulation of the middle cerebral artery branches in the sylvian fissure. However, injury to the anterior choroidal artery is a potential risk with resection of the mesial structures. ${ }^{9}$ Our single case of monoparesis was most likely the result of an embolic distal branch occlusion. To prevent injury to the vasculature, it is essential to minimize brain retraction and manipulation and to preserve the pial plane against the brainstem and surrounding structures. We did not see any case of transient hemiparesis, which has been described as a complication of this procedure.,13 Less commonly, dysphasia has been documented after resection of the dominant temporal lobe. ${ }^{15}$ However, no permanent aphasia was reported after our approach. Nevertheless, occasional transient dysphasia is seen after resection of the dominant temporal lobe, but it usually resolves within 48 hours. The most likely explanation for the lack of permanent aphasia is the limited neocortical resection with this technique. Ojemann et al. elegantly demonstrated that individual variability in language localization among patients might occur; but more significantly, in their study, none of the language centers were localized within the ITG. ${ }^{11}$ This advantage allows the ITG approach to be used for resection of dominant and nondominant temporal lobes.

Visual field defects, particularly superior quadrant defects, might occur after temporal lobe resection. Major 
quadrantanopia has been documented for $2 \%-4 \%$ of patients. ${ }^{3,13}$ This complication can result from injury to the Meyer loop as it courses through the roof of the lateral ventricle. A major limitation of this study, and most of the literature, is the lack of formal visual field testing. The healthcare industry limits this type of evaluation in the absence of clinically significant symptoms. All patients reported here underwent a detailed neurological assessment, and no major visual field defects were detected to confrontation. We understand that minor, nonclinical significant injury to the Meyer loop might occur in this patient population. To minimize this complication, we recommend approaching the ventricle anteriorly and through the floor. In addition, limiting the extent of the ventricular opening will result in fewer complications. Palsy of the third cranial nerve is another uncommon complication of mesial temporal lobe resection (reportedly less than $1 \%$ ). ${ }^{3}$ This complication most likely results from transpial manipulation of the nerve. Fortunately, we did not see this complication during the follow-up period.

The most common complication in our series was development of subacute and chronic subdural hematoma. Evacuation of symptomatic subdural fluid collection was needed by 3 patients because of persistent mass effect. No permanent complications resulted from this intervention. Early in our experience, we hypothesized that liberal use of mannitol and aggressive hyperventilation resulted in higher incidence of this problem. However, ventricular opening has been associated with distant hemorrhagic complications. ${ }^{5,26}$ Stretching of the subdural veins is the most likely explanation. Risk is higher for patients older than 40 years who undergo mesial temporal lobe resection, although our results are not statistically significant $(\mathrm{p}=0.193)$. In addition, for 1 patient, delayed intracranial hemorrhage developed within the surgical cavity; the patient was readmitted to the intensive care unit for observation and no further intervention was required. No long-term morbidity resulted from this complication. No cases of transient cerebral edema were documented. Epidural hemorrhages also have been described ${ }^{18}$; however, we did not identify any case with significant epidural collection, probably because our technique uses a small bone flap and minimal epidural dissection to access the mesial temporal lobe structures. In addition, we did not identify any cases with postoperative CSF leakage, which might be explained by the small craniotomy size, meticulous dural closure (with occasional use of dural sealant), and detailed approximation of the temporalis muscle.

The most common epilepsy surgery complication reported in the literature is wound infection. ${ }^{3,18}$ Among the patients reported here, 2 had superficial wound infections; 1 required wound revision and both required prolonged intravenous antibiotic therapy. We routinely give prophylactic intravenous antibiotics for 24 hours, but other authors do not use them regularly; with regard to antibiotic prophylaxis, complications do not differ significantly. ${ }^{18}$

Transient frontalis nerve palsy developed in 1 patient in our series. The most plausible explanation is aggressive dissection and retraction of the soft tissue during the initial exposure. This cosmetic complication can be eliminated by attention to detail and recognition of the location of the frontalis nerve branch. For the patient reported here, the nerve palsy resolved within 6 weeks with no long-term morbidity.

Our results demonstrate that this surgery is safe and effective but that complications do occur, especially in older patients. Our findings emphasize the importance of early referral for surgical treatment for patients with medically refractory epilepsy. Complications after temporal lobe resection for epilepsy are few. ${ }^{10}$ Our results support the notion that sex, race, and income are not predictive of postsurgical complications. ${ }^{10}$ In addition, complications did not differ significantly according to histopathology or side of surgery. Nevertheless, for minimizing postsurgical complications, detailed knowledge of microsurgical anatomy is imperative. ${ }^{24}$

We acknowledge the limitations of a single-center, retrospective study (such as unblinded evaluators) and therefore recommend further studies to corroborate our findings. In addition, postoperative formal visual field testing would provide useful information, but current healthcare limitations prevent its being performed on a consistent basis. Neuropsychological complications, which might substantially affect quality of life, are not addressed in detail in this article. This series represents a fairly homogeneous group of patients with MTLE who underwent selective transcortical resection of the mesial temporal lobe. These data can be useful for comparison as new alternative techniques for the treatment of medically refractory MTLE become more accessible for this population.

\section{Conclusions}

Epilepsy surgery is safe and effective. Mortality and morbidity rates after epilepsy surgery are lower than those after other common neurosurgical procedures. Advances in operative techniques, anesthesia, and perioperative management have decreased the risk for complications. Nevertheless, postsurgical complications can occur. Risks and benefits should be quantified and shared with patients before surgery. However, because complications are more common among older patients, patients with medically refractory epilepsy should be referred for surgical treatment early. Obviously, detailed knowledge of the microsurgical anatomy and surgical experience play a major role in minimizing morbidity in this group of patients.

\section{Disclosure}

The authors report no conflict of interest concerning the materials or methods used in this study or the findings specified in this paper.

Author contributions to the study and manuscript preparation include the following. Conception and design: Vale. Acquisition of data: all authors. Analysis and interpretation of data: Reintjes, Garcia. Drafting the article: all authors. Critically revising the article: Vale. Reviewed submitted version of manuscript: Vale. Approved the final version of the manuscript on behalf of all authors: Vale. Study supervision: Vale.

\section{Acknowledgment}

The authors thank Jenna Dionisio, M.P.H., for the statistical analysis. 


\section{References}

1. Barbaro NM, Quigg M, Broshek DK, Ward MM, Lamborn KR, Laxer KD, et al: A multicenter, prospective pilot study of gamma knife radiosurgery for mesial temporal lobe epilepsy: seizure response, adverse events, and verbal memory. Ann Neurol 65:167-175, 2009

2. Bartolomei F, Hayashi M, Tamura M, Rey M, Fischer C, Chauvel $\mathrm{P}$, et al: Long-term efficacy of gamma knife radiosurgery in mesial temporal lobe epilepsy. Neurology 70:1658-1663, 2008

3. Behrens E, Schramm J, Zentner J, König R: Surgical and neurological complications in a series of 708 epilepsy surgery procedures. Neurosurgery 41:1-10, 1997

4. Benbadis SR, Heriaud L, Tatum WO, Vale FL: Epilepsy surgery, delays and referral patterns - are all your epilepsy patients controlled? Seizure 12:167-170, 2003

5. Clusmann H, Kral T, Marin G, Van Roost D, Swamy K, Schramm J: Characterization of hemorrhagic complications after surgery for temporal lobe epilepsy. Zentralbl Neurochir 65:128-134, 2004

6. Curry DJ, Gowda A, McNichols RJ, Wilfong AA: MR-guided stereotactic laser ablation of epileptogenic foci in children. Epilepsy Behav 24:408-414, 2012

7. Duckworth E, Vale FL: Trephine epilepsy surgery: the inferior temporal gyrus approach. Neurosurg 63 (1 Suppl 1): ONS156-ONS161, 2008

8. Gilioli I, Vignoli A, Visani E, Casazza M, Canafoglia L, Chiesa V, et al: Focal epilepsies in adult patients attending two epilepsy centers: classification of drug-resistance, assessment of risk factors, and usefulness of "new" antiepileptic drugs. Epilepsia 53:733-740, 2012

9. Helgason C, Caplan LR, Goodwin J, Hedges T III: Anterior choroidal artery-territory infarction. Report of cases and review. Arch Neurol 43:681-686, 1986

10. McClelland S III, Guo H, Okuyemi KS: Population-based analysis of morbidity and mortality following surgery for intractable temporal lobe epilepsy in the United States. Arch Neurol 68:725-729, 2011

11. Ojemann G, Ojemann J, Lettich E, Berger M: Cortical language localization in left, dominant hemisphere. An electrical stimulation mapping investigation in 117 patients. J Neurosurg 71: 316-326, 1989

12. Ramos E, Benbadis S, Vale FL: Failure of temporal lobe resection for epilepsy in patients with mesial temporal sclerosis: results and treatment options. Clinical article. J Neurosurg 110: 1127-1134, 2009

13. Rydenhag B, Silander HC: Complications of epilepsy surgery after 654 procedures in Sweden, September 1990-1995: a multicenter study based on the Swedish National Epilepsy Surgery Register. Neurosurgery 49:51-56, 2001

14. Salanova V, Markand O, Worth R: Temporal lobe epilepsy surgery: outcome, complications, and late mortality rate in 215 patients. Epilepsia 43:170-174, 2002

15. Schramm J, Aliashkevich AF: Surgery for temporal mediobasal tumors: experience based on a series of 235 patients. Neurosurgery 62 (6 Suppl 3): 1272-1282, 2008

16. Sindou M, Guenot $M$, Isnard J, Ryvlin $P$, Fischer $C$, Mauguière F: Temporo-mesial epilepsy surgery: outcome and complications in 100 consecutive adult patients. Acta Neurochir (Wien) 148:39-45, 2006

17. Spencer DD, Spencer SS, Mattson RH, Williamson PD, Novelly RA: Access to the posterior medial temporal lobe structures in the surgical treatment of temporal lobe epilepsy. Neurosurgery 15:667-671, 1984

18. Tanriverdi T, Ajlan A, Poulin N, Olivier A: Morbidity in epilepsy surgery: an experience based on 2449 epilepsy surgery procedures from a single institution. Clinical article. J Neurosurg 110:1111-1123, 2009

19. Tanriverdi T, Olivier A, Poulin N, Andermann F, Dubeau F: Long-term seizure outcome after mesial temporal lobe epilepsy surgery: corticalamygdalohippocampectomy versus selective amygdalohippocampectomy. J Neurosurg 108:517-524, 2008

20. Tatum WO IV, Benbadis SR, Vale FL: The neurosurgical treatment of epilepsy. Arch Fam Med 9:1142-1147, 2000

21. Uribe JS, Vale FL: Limited access inferior temporal gyrus approach to mesial basal temporal lobe tumors. Clinical article. J Neurosurg 110:137-146, 2009

22. Vale FL, Effio E, Arredondo N, Bozorg A, Wong K, Martinez $C$, et al: Efficacy of temporal lobe surgery for epilepsy in patients with negative MRI for mesial temporal lobe sclerosis. J Clin Neurosci 19:101-106, 2012

23. Vale FL, Pollock G, Benbadis SR: Failed epilepsy surgery for mesial temporal lobe sclerosis: a review of the pathophysiology. Neurosurg Focus 32(3):E9, 2012

24. Wen HT, Rhoton AL Jr, de Oliveira E, Cardoso AC, Tedeschi $\mathrm{H}$, Baccanelli M, et al: Microsurgical anatomy of the temporal lobe: part 1: mesial temporal lobe anatomy and its vascular relationships as applied to amygdalohippocampectomy. Neurosurgery 45:549-592, 1999

25. Wiebe S, Blume WT, Girvin JP, Eliasziw M: A randomized, controlled trial of surgery for temporal-lobe epilepsy. $\mathbf{N}$ Engl J Med 345:311-318, 2001

26. Ziyal I, Bilginer B, Yavuz K, Turk C, Ozgur C, Benli K: Does ventricular opening promote remote cerebellar haemorrhage? Turk Neurosurg 22:102-104, 2012

\section{Manuscript submitted February 12, 2013.}

Accepted March 21, 2013.

Please include this information when citing this paper: DOI: 10.3171/2013.3.FOCUS1354.

Address correspondence to: Fernando L. Vale, M.D., Department of Neurosurgery and Brain Repair, University of South Florida, 2 Tampa General Circle, 7th Floor, Tampa, Florida 33606. email: fvale@health.usf.edu. 